\title{
Thalamocortical Angular Tuning Domains within Individual Barrels of Rat Somatosensory Cortex
}

\author{
Randy M. Bruno, ${ }^{1}$ Vivek Khatri, ${ }^{2}$ Peter W. Land, ${ }^{2}$ and Daniel J. Simons ${ }^{2}$ \\ ${ }^{1}$ Department of Cell Physiology, Max Planck Institute for Medical Research, 69120 Heidelberg, Germany, and ${ }^{2}$ Department of Neurobiology, University of \\ Pittsburgh School of Medicine, Pittsburgh, Pennsylvania 15261
}

In the rodent somatosensory cortex, whisker-related barrels in layer IV are morphological counterparts of functional cortical columns that extend throughout the cortical depth. We used microelectrode recordings and spike-triggered averaging of field potentials evoked by single thalamic barreloid neurons to investigate functional thalamocortical microcircuits. The function of such circuits was probed by deflecting the principal whisker of a barrel in different angular directions. We found that individual barrels contain minicolumns of neurons preferring the same deflection angle. Angular tuning domains are established by convergent inputs from thalamocortical cells with corresponding angular preferences. Processing within such domains may depend on local connectivity among vertically aligned barrel neurons.

Key words: minicolumn; somatosensory; whisker; local circuit; thalamocortical; barrel

\section{Introduction}

Seminal studies of cat and monkey primary somatosensory cortex (S1) first established that the neocortex is organized into vertically oriented modules, called functional cortical columns, that form the fundamental building blocks of cortical information processing (Mountcastle, 1957; Powell and Mountcastle, 1959). Functional cortical columns have been described subsequently in a wide variety of cortical areas and species. Microcircuitry within cortical columns preserves the representation of specific stimulus features, such as location on the sensory receptor surface, and allows for the elaboration of new, related receptive field properties (Hubel and Wiesel, 1977). Early studies of rodent S1 established the whisker-related barrel as a morphological counterpart in layer IV of a functional column extending throughout the depths of the cortex (see also Armstrong-James, 1975; Durham and Woolsey, 1977; Simons, 1978; Simons and Woolsey, 1979). These and subsequent studies have identified important commonalities in the functional organization of sensory cortices in various modalities and species (Jones and Diamond, 1995). Barrels, readily identified in histological sections and living cortical slices, are particularly well suited for investigations of the microcircuitry underlying columnar function.

Functional cortical columns are vertically oriented cylinders with diameters of 300-500 $\mu \mathrm{m}$. Substantial evidence exists that cortical columns are assembled from collections of smaller anatomical and/or functional subdivisions, called minicolumns, with diameters of $<100 \mu \mathrm{m}$ (for review, see Mountcastle, 2003). In sensory cortical maps, minicolumns may permit the represen-

\footnotetext{
Received June 5, 2003; revised Sept. 3, 2003; accepted Sept. 3, 2003.

This work was supported by National Institutes of Health Grants NS19950 and NS 41428. We thank H. Kyriazi for expert technical assistance and A. Myers for histology.

Correspondence should be addressed to Dr. Randy M. Bruno, Department of Cell Physiology, Max Planck Institute for Medical Research, Jahnstrasse 29,69120 Heidelberg, Germany. E-mail: bruno@mpimf-heidelberg.mpg.de. Copyright $\odot 2003$ Society for Neuroscience $\quad$ 0270-6474/03/239565-10\$15.00/0
}

tation of multiple stimulus attributes associated with a circumscribed region of the receptor periphery. Whisker-related barrels are $\sim 400 \mu \mathrm{m}$ in diameter, and barrel centers are heterogeneous with respect to a number of anatomical and physiological markers (Land and Simons, 1985; McCasland and Woolsey, 1988; Land et al., 1995; Land and Akhtar, 1999). These observations raise the possibility that barrels also contain minicolumns or analogous vertically oriented operational units.

A conspicuous feature of the whisker-to-barrel system is that individual neurons respond differently depending on the angular direction in which the whisker is displaced from its resting position. Angular tuning, present in trigeminal ganglion cells (Lichtenstein et al., 1990), is thought to be caused by the selective circumferential innervation of a sinus-hair follicle by a given primary afferent axon. Angular sensitivity has not been investigated in behavioral studies; thus, its behavioral relevance remains unclear. However, its pervasive presence throughout the whisker/ barrel system indicates robust mechanisms for preserving this property across synaptic stations (Simons and Carvell, 1989). Angular tuning is observed in cortical neurons despite substantial anatomical divergence and convergence of subcortical and intracortical pathways, especially prominent in the thalamocortical system.

Here, we probed the micro-organization of thalamocortical circuitry within layer IV barrels by deflecting the principal whisker $(\mathrm{PW})$ in different angular directions. Using microelectrode recordings and spike-triggered averaging of field potentials evoked by single thalamocortical neurons, we found that an individual barrel is composed of multiple angular tuning domains, each receiving convergent inputs from thalamic neurons whose angular preferences are similar to each other and to the preferred angle of nearby, vertically aligned barrel neurons. These findings suggest the existence, within individual barrels, of collections of functional minicolumns based on angular preference. 


\section{Materials and Methods}

Spike-triggered averaging data were collected in five female rats weighing 275-350 gm (Sprague Dawley strain; Hill Top Lab Animals, Scottsdale, PA). Surgical preparation and maintenance of the rats during the recording sessions are similar to those described in detail by Simons and Carvell (1989) and most recently by Bruno and Simons (2002). Briefly, rats were initially anesthetized with halothane $(1.5-2.0 \%)$. Bone overlying the right barrel cortex $\left(<0.7 \mathrm{~mm}^{2}\right)$ and the ventral posterior medial nucleus (VPM) of the thalamus $\left(<2 \mathrm{~mm}^{2}\right)$ was removed. For neural recordings, halothane was discontinued, and the rat was maintained in a lightly narcotized, sedated state by the intravenous infusion of fentanyl (Sublimaze; Janssen Pharmaceuticals, Janssen Biochimica, Berse, Belgium) $\left(\sim 10 \mu \mathrm{g} \cdot \mathrm{kg}^{-1} \cdot \mathrm{hr}^{-1}\right)$. To prevent spontaneous whisker movement, which would preclude the use of our whisker stimulators (below), neuromuscular blockade was induced with pancuronium bromide (1.6 $\mathrm{mg} \cdot \mathrm{kg}^{-1} \cdot \mathrm{hr}^{-1}$ ), and the animal was artificially respired ( $\sim 90$ breaths/ $\mathrm{min}$ ) using a positive-pressure ventilator. The body temperature was maintained at $37^{\circ} \mathrm{C}$ by a servo-controlled heating blanket (Harvard Apparatus, Holliston, MA). A computer running custom-written software continuously monitored the rat's electroencephalogram, mean arterial pressure, arterial pulse rate, and tracheal airway pressure. Experiments were terminated if any of the above indicators could not be maintained within the normal physiological range. At the termination of the experiment, the rat was deeply anesthetized with sodium pentobarbital and perfused transcardially for subsequent histological evaluation of recording sites.

Spike-triggered averages. We used averaged cortical local field potentials (LFPs) triggered from thalamic spikes to infer the functional innervation of a barrel by individual thalamocortical (TC) neurons. Spiketriggered averages (STAs) were obtained by calculating the mean cortical LFPs at the time of spontaneous discharges $(n>1000)$ of individual thalamic neurons. LFPs were recorded within a barrel using a concentric array of two to four extracellular electrodes horizontally spaced $\sim 100-$ $150 \mu \mathrm{m}$ tip to tip. The spontaneous activity of a single TC neuron was simultaneously recorded using an extracellular electrode located in the somatotopically aligned thalamic barreloid. For each recorded TC neuron, LFPs were obtained simultaneously and averaged separately for each of two to four different locations within the barrel. Absolute voltage measurements of LFPs were checked for accuracy by inserting the electrode array into a saline bath and passing current via an Omnical 2001 Voltage Calibrator (World Precision Instruments, Sarasota, FL) connected in series.

Extracellular recordings were made using 2-6 M $\Omega$ electrodes made from pulled and beveled quartz-insulated platinum-tungsten ( $90-10 \%)$ core fibers (Uwe Thomas Recording, Giessen, Germany). Thalamic electrodes were slowly advanced perpendicular to the pial surface using a hydraulic microdrive (David Kopf Instruments, Tujunga, CA), and cortical recordings were obtained with a multi-electrode array microdrive (Uwe Thomas Recording) used to independently control two to four recording electrodes. Signals were amplified by conventional means. Single- and multi-unit activities were bandpass-filtered between $300 \mathrm{~Hz}$ and $10 \mathrm{kHz}$, and LFPs between $10 \mathrm{~Hz}$ and $10 \mathrm{kHz}$. All analog signals were digitized at $32 \mathrm{kHz}$ using a $1 \mathrm{GHz}$ personal computer equipped with a PCI-MIO-16E-1 board (National Instruments, Austin, TX) and running custom data acquisition software written in LabVIEW, Visual Basic (Microsoft, Seattle, WA). Recording sites and/or the termination of electrode tracks were marked using small electrolytic lesions.

Single-unit and multi-unit waveform samples exceeding amplitude thresholds were parsed from the continuous signals, displayed, and stored to disk along with the timestamp and trial information. Multi-unit recordings were used to assess the angular tuning of small groups of nearby cortical units. For thalamic recordings, data were obtained only from well-isolated single units. Typical signal/noise ratios were on the order of 10/1, and recordings consisted of only a single well-isolated unit; occasionally, two readily discriminable units were present on a recording channel. Even with the best-isolated waveforms, we used spike sorting techniques to further ensure the quality of single-unit isolation. Spike sorting was performed offline using MCLUST version 2.0 (A. David
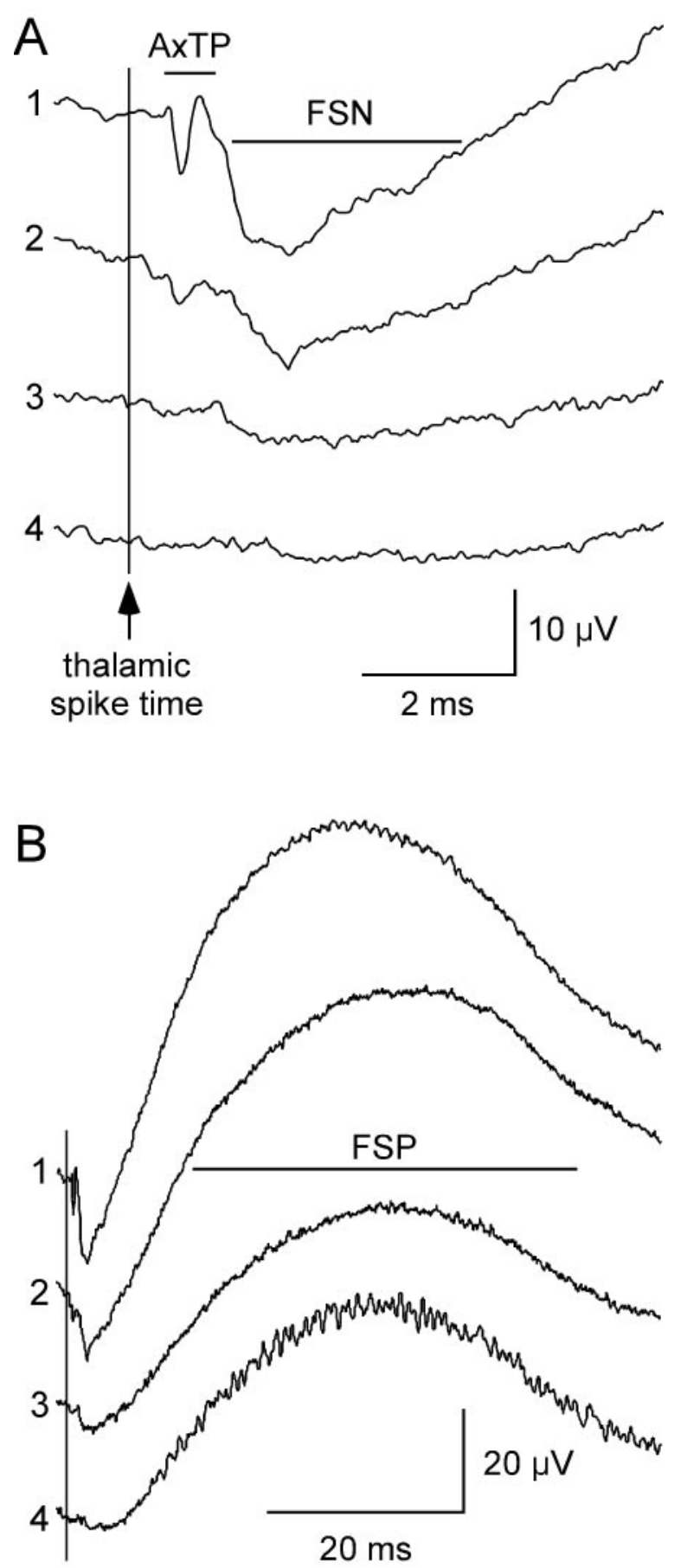

Figure 1. STAs of LFPs recorded in cortical layer IV reveal three distinct responses to spikes of a single thalamic neuron. A, STAs generated at four different sites in a cortical barrel by a single TC neuron. The AxTP reflects presynaptic afferent activity of the TC neuron. The FSN reflects the initial postsynaptic cortical response evoked by the afferent. The vertical line indicates the time of thalamic spikes. B, Expanded time scale shows the FSP that follows the FSN.

Redish, University of Minnesota, Minneapolis, MN) and our own custom-written C programs (Bruno and Simons, 2002). LFPs were continuously streamed to disk without parsing.

STAs have 1-3 distinct components, which we describe using the terminology of Swadlow and Gusev (2000), who first recorded them in the barrel cortex. The earliest component, the axon terminal potential (AxTP), is a rapid biphasic or triphasic response occurring $<1 \mathrm{msec}$ after the discharge of the thalamic spike (Fig. 1A) and just before the earliest spiking of cortical barrel neurons (Kyriazi et al., 1994). A longer-duration 
Table 1. Measurements of components of spike-triggered averages for which AxTP $\geq 1 \mu \mathrm{V}$

\begin{tabular}{llcc}
\hline & Amplitude $(\mu \mathrm{V})$ & $\begin{array}{l}\text { Duration } \\
(\mathrm{msec})\end{array}$ & Latency (msec) \\
\hline AxTP & $2.0 \pm 1.1$ & $0.69 \pm 0.13$ & $0.60 \pm 0.27$ \\
FSN & $3.1 \pm 2.1$ & $4.9 \pm 3.3$ & $1.3 \pm 0.44$ \\
FSP & $9.5 \pm 9.2$ & & \\
\hline
\end{tabular}

Values are means \pm SD. AxTP and FSN amplitudes are measured from baseline (just prior to thalamic spike) to negative peak, and FSP amplitude from baseline to positive peak.

negative potential, termed the focal synaptic negativity (FSN), typically occurs at a latency of 1-2 msec (Fig. 1A). As in previous studies in awake rabbits (Swadlow and Gusev, 2000), we found that these two components sometimes overlap partially. The largest component is the lateoccurring focal synaptic positivity (FSP) (Fig. $1 B$ ). All three components are maximal in layer IV (Swadlow and Gusev, 2000), and current source density analysis has further demonstrated that current sinks are usually predominant in or even restricted to layer IV (Swadlow et al., 2002). Therefore, these signals likely represent local excitatory and inhibitory events. The AxTP in particular is thought to reflect the discharge of the presynaptic arbor of the thalamic neuron because: (1) its latency is tightly correlated with that of thalamic spikes elicited by antidromic activation and (2) pharmacological blockade of glutamatergic synaptic transmission by DNQX abolishes the FSN and FSP but does not change the AxTP (Swadlow and Gusev, 2000; Swadlow et al., 2002). Therefore, we used the AxTP as an indirect measure of the number of nearby active synapses made by a TC neuron, whereas the FSN and FSP served as measures of initial excitatory and subsequent inhibitory postsynaptic cortical responses, respectively. Latency, duration, and amplitude measurements of these components are given in Table 1 and are consistent with previous reports.

Single-unit cortical recording. Basic response properties of a large part of the barrel single-unit data were described previously by Simons and Carvell (1989). Units were recorded using double-barrel glass micropipettes [ $4-8 \mathrm{M} \Omega$, one barrel of which contained $3 \mathrm{M} \mathrm{NaCl}$ and the other $2 \% \mathrm{w} / \mathrm{v}$ horseradish peroxidase (HRP) for marking electrode tracks and specific recording sites] (Simons and Land, 1987). An amplitude discriminator (World Precision Instruments) was used to digitize spikeevent times, which were saved on an LSI 11 computer (Digital Equipment Corp., Maynard, MA). Preliminary mapping penetrations and scaled drawings of the surface vasculature were used to target barrel centers, subsequently confirmed histologically (see below). For data collection in vertical microelectrode penetrations, we were careful to orient the electrode path perpendicular to the pial surface, and we attempted to isolate single units at several depths within the barrel. Only one barrel was studied per experiment.

In two experiments the recording microelectrode was oriented tangential to the pial surface and parallel to layer IV, permitting us to sample activity in adjacent barrels. A barrel corresponding to one of the caudal whiskers (e.g., C2) was first identified using vertical penetrations. The microelectrode was positioned so that it entered the cortex in a trajectory parallel to the cortical surface, beginning several millimeters posterolateral and $\sim 1 \mathrm{~mm}$ deep to the pial surface above the physiologically identified barrel. In each experiment, three parallel microelectrode penetrations were made, passing from barrels representing dorsal whiskers to those representing more ventral whiskers. In this plane at the medial aspect of the barrel field, microelectrodes can remain in layer IV across at least two adjacent barrels (see Fig. 5 in Land and Simons, 1985).

Angular tuning data are analyzed only for regular-spike (presumed excitatory) barrel units, because fast-spike units (presumed inhibitory barrel neurons) typically respond equivalently strongly to all, or most, deflection angles (Simons and Carvell, 1989; Bruno and Simons, 2002). Analyses are based on 250 units recorded in 76 vertical penetrations in 16 experiments. At least two single units were isolated per penetration. Thirty-nine regular-spike units were recorded in six tangential penetrations in two experiments.

Vibrissa stimulation. The vibrissa that elicited the strongest responses at successive recording locations was defined as the principal whisker. Piezo- electric stimulators (Simons, 1983), attached 10-12 mm from the base of the hair, were used to apply ramp-and-hold deflections (Simons and Carvell, 1989). The whisker was deflected randomly in each of eight cardinal directions in $45^{\circ}$ increments relative to the horizontal alignment of the whisker rows. Ten or 20 blocks of such stimuli were delivered to the PW.

Histology. The cortex was cut tangentially in $60 \mu \mathrm{m}$ frozen serial sections, reacted for cytochrome oxidase, and counterstained with thionine. The thalamus was processed similarly, except that the tissue was sectioned in the coronal plane. For experiments using HRP to mark electrode tracks, sections were further postfixed for several minutes in $2 \%$ gluteraldehyde in $0.1 \mathrm{M}$ phosphate buffer, which limited HRP enzymatic activity to the vicinity of the ejection site. Adjacent sections then were stained for HRP according to Adams (1981) or for CO (Land and Simons, 1985); $10 \%$ catalase was included in the CO reaction mixture to attenuate HRP cross-reactivity with the diaminobenzidine chromogen (Simons and Land, 1987). During the recording experiment, the locations of individual electrode penetrations were carefully noted on scaled drawings of the surface vasculature, which subsequently could be aligned with the most superficial histological sections. Using the scaled drawings, microdrive readings, signs of tissue disruption around electrode tracks, and either HRP spots or electrolytic lesions, recording sites were localized with respect to individual cortical barrels. We report here only data obtained within the CO-rich barrel center, which in our hands has consistently been found to correspond to microdrive depth readings of $700-$ $1000 \mu \mathrm{m}$ (Kyriazi et al., 1996). We were unable to correlate unit properties with the distribution of $\mathrm{CO}$ staining within barrels (for example, $\mathrm{CO}$ blotches) because such subtle histochemical staining patterns are especially difficult to visualize after disruption of the microvasculature by multiple microelectrode penetrations. For thalamic recordings, sections were examined to verify the presence of electrode tracks (or marker lesions) in the dorsal portion of the VPM, where the barreloids are located.

Statistical analysis. Unit responses were quantified by measuring the average number of spikes per stimulus during a 20 msec period immediately after the onset or offset of whisker deflection (i.e., ON and OFF responses) (Simons and Carvell, 1989). The angular tuning of each neuron was quantified by constructing a polar plot of the ON response for each of the eight deflection angles. Pairs of polar plots (e.g., from successively recorded units) were compared using a similarity index (SI), calculated as a standard statistical correlation coefficient of the corresponding $\mathrm{ON}$ responses of the two units to each of the eight deflection angles:

$$
\mathrm{SI}=\frac{1}{n-1} \sum_{i=1}^{n}\left(\frac{x_{i}-\bar{x}}{s_{x}}\right)\left(\frac{y_{i}-\bar{y}}{s_{y}}\right),
$$

where $x_{i}$ is the $\mathrm{ON}$ response of cell $X$ to deflections at angle $i, y_{i}$ is the $\mathrm{ON}$ response of cell $Y$ to deflections at angle $i, \bar{x}$ and $\bar{y}$ are the respective means over all eight angles, $s_{x}$ and $s_{y}$ are the SDs, and $n=8$. Therefore, a correlation coefficient of 1.0 indicates equivalently shaped polar plots, and a value of -1.0 indicates complete dissimilarity of shape. We also analyzed angular sensitivity by computing for each polar plot a mean vector whose phase angle represents the overall directional preference of the unit and whose magnitude is proportional to the strength of tuning.

Data were analyzed using a combination of programs written in LabVIEW, Visual Basic (Microsoft), and Splus 2000 (Insightful Corp., Seattle, WA). Distributions were compared using the nonparametric Wilcoxon rank-sum (Mann-Whitney) test.

\section{Results}

\section{Functional innervation of barrels by thalamic neurons}

We assessed the functional impact of a TC neuron within small regions of its homologous barrel by measuring LFPs generated as a result of the spontaneous spikes of the neuron. Using spiketriggered averages, we found that the functional impact of a thalamic neuron is different at various locations within a barrel. Figure $1 A$ shows spike-triggered averages obtained from a single $\mathrm{TC}$ neuron at four recording sites within the same barrel. The 
neuron evoked its largest and most distinct AxTP and FSN at site 1. A smaller AxTP and FSN were observed at site 2. Although neither site 3 nor site 4 exhibited obvious terminal potentials, a small FSN was detected at least for site 3 . We interpret this set of averages to indicate that the associated thalamic neuron had a large number of functional synapses in the immediate vicinity of site 1 , a smaller number at site 2 , and few or no synapses at sites 3 and 4. FSPs were large at all cortical sites, even when the FSN was almost entirely absent (Fig. $1 B$, site 4 ). Spike-triggered averages from four additional example TC neurons are shown in Figure 3. For all 28 TC neurons studied, the presence and/or size of AxTPs and FSNs differed among recording locations within a barrel. These findings suggest that the functional impact of individual TC neurons is not uniform throughout the barrel (see also Swadlow et al., 2002, for laminar variability).

\section{TC inputs and cortical angular selectivity}

Each thalamic and cortical neuron exhibits a preference for the angular direction of whisker movement, and individual thalamic neurons appear to affect some regions of a barrel more strongly than others. Therefore, we compared the angular preference of a thalamic neuron to the angular preference of cortical neurons in the particular region(s) of the barrel that the thalamic axon innervates, as judged by the amplitude of its AxTP. We recorded both thalamic spike-triggered averages and multi-unit activity at three depths throughout layer IV (750, 850, and $950 \mu \mathrm{m})$ at four cortical sites in each of two animals. Polar plots were created for the thalamic unit and for the cortical multi-unit activity. The angular tuning preferences of multi-unit activity were similar at different depths within a penetration (see below). Likewise, we found no systematic effect of depth on the AxTPs and FSNs of thalamic spike-triggered averages.

The similarity index of the polar plots for a TC neuron and multi-unit activity at a cortical site was plotted against the amplitude of the terminal potentials evoked at that location by the thalamic cell ( $n=7$ cells). As shown in Figure $2 A$, the terminal potential at a given site was small or nonexistent if the thalamic and cortical polar plots were oppositely tuned (SIs less than -0.5). The largest AxTPs were generated at sites where the tuning profiles were similar (SIs $\geq 0.5$ ). The relationship of tuning similarity to AxTP amplitude was best fit by logarithmic regression $\left(r^{2}=0.55\right)$ and was highly statistically significant $(p<0.0001)$. Limiting the analysis to activity recorded at any one depth (e.g., $750,850$, or $950 \mu \mathrm{m})$ yielded equivalent results $\left(r^{2}=0.6\right.$; $p<$ 0.0001; data not shown). A similar logarithmic relationship exists for the FSN $\left(r^{2}=0.32 ; p<0.0001\right)$ (Fig. $2 B$ ). The poorer fit indicates that the FSN is less tightly coupled than the AxTP to the tuning similarity of the thalamic and cortical neurons.

\section{Angular selectivity of convergent thalamic inputs}

We have shown that a TC neuron with a particular preferred angle produces a larger AxTP in a barrel region in which neurons are similarly tuned to the TC neuron. This suggests the possibility that TC neurons with similar angular preferences converge onto a common cortical site. Therefore, we compared the angular tuning of pairs of thalamic neurons with the AxTPs they produced at different locations within a barrel. The two TC neurons shown in Figure $3 A$ had similar directional profiles $(S I=0.43)$. In both cases, AxTP amplitudes relative to baseline were maximal at site 2 and smaller at site 1 . Figure $3 B$ shows data for two other thalamic neurons, recorded in another rat, that generated different-sized STAs at each barrel site. The polar plots for these thalamic neurons were oppositely tuned, as indicated by a negative SI $(-0.89)$.
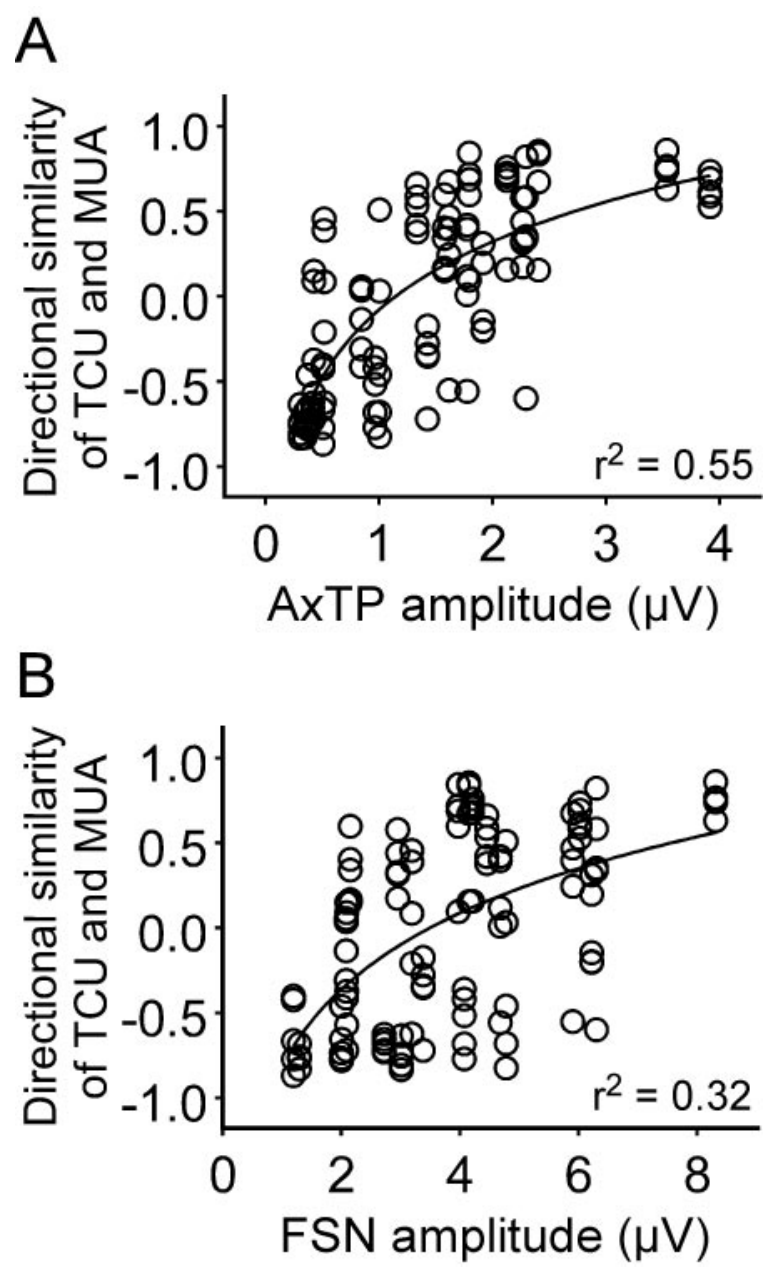

Figure 2. TC contributions to angular tuning domains. A, The SI comparing the polar plot of individual TC neurons to the polar plot of multi-unit activity recorded at a cortical site is proportional to the amplitude of the AxTP produced by the TC neuron at that location in the barrel. Line, Fit of logarithmic (base 10) regression $\left(r^{2}=0.55 ; p<0.0001\right)$. $B$, Same analysis as in $A$, but for the FSN $\left(r^{2}=0.32 ; p<0.0001\right)$.

Specifically, the TC neuron depicted in the top panel preferred ventral whisker deflections and produced a large AxTP at site 2 but virtually no detectable AxTP at site 1. The less-well-tuned TC neuron (Fig. 3, bottom) preferred dorsal deflections and produced its larger AxTP at site 1 and a barely detectable response at site 2 .

We quantified TC convergence by calculating the amplitude differences of AxTPs generated at each of the two to four cortical recording sites for all 63 possible combinations of TC neurons recorded within the same experiments (238 total site comparisons). Smaller differences in AxTP amplitudes evoked by pairs of TC neurons were considered to reflect greater overlap of their arbors or absence of significant termination by both axons. Differences in AxTP amplitude were compared with angular similarity indices calculated for pairs of TC neurons (Fig. 4). We found that the similarity of TC angular tuning was related to the relative sizes of the AxTPs in the cortical barrel. The degree of match between terminal potentials was significantly different ( $p=0.0012$; Wilcoxon) between dissimilar (SI less than -0.5 ) and similar TC pairs $(S I \geq 0.5)$. Dissimilar pairs also differed from those with intermediate SIs $(-0.5 \leq \mathrm{SI}<0.0$ and $0.0 \leq \mathrm{SI}<0.5$; $p=0.004$ and $p=0.0001$, respectively). Thus, similarly tuned TC pairs have overlapping domains of effective synapses within the 

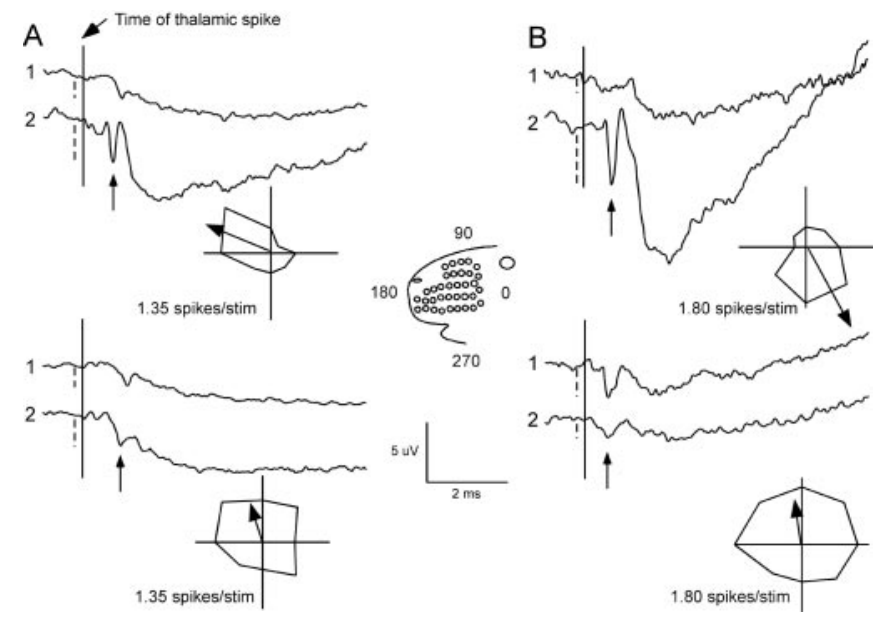

Figure 3. Different thalamic neurons generate different STAs at any given cortical site. A, STAs and polar plots for two TC neurons, recorded in the same thalamic barreloid, with moderately similar polar plots $(S I=0.43)$. Polar plots show average responses, in spikes per stimulus, to eight deflection angles (see inset for orientation); both neurons prefer rostrodorsal deflections (vector arrows in polar plots) and produce the larger of their AxTPs at cortical recording site 2 (arrows). Dashed vertical lines indicate the AxTP peak amplitude relative to baseline. B, STAs and polar plots for two $\mathrm{TC}$ neurons, recorded in a different animal, with different angular tuning $(\mathrm{SI}=-0.89)$. Note that the TC neuron in the top panel prefers ventral deflections and produces a large AxTP at site 2, whereas the oppositely tuned TC neuron of the bottom panel produces little AxTP at the same location.

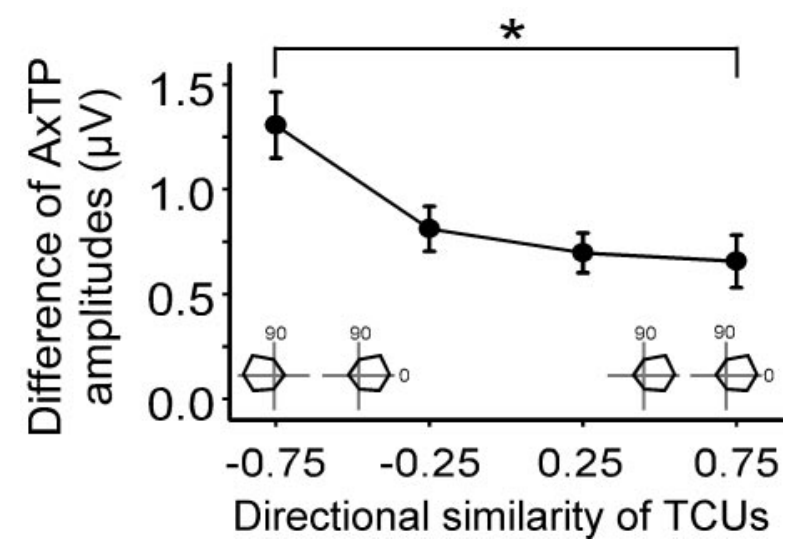

Figure 4. Convergence of TC neurons with similar or opposite tuning. $x$-axis, SI of pairs of TC neurons recorded in the same experiment with schematic examples of anticorrelated (left inset) and correlated (right inset) response profiles ( 0 , caudal deflections, 90 , dorsal deflections). $y$-axis, Absolute value of the difference in AxTP amplitudes for the TC pairs. Data are plotted as means \pm SEM. Oppositely tuned $(S I<-0.5)$ pairs have larger differences in their terminal potentials than similarly tuned $(S \mid>0.5)$ ones $\left({ }^{*} p=0.0001\right)$.

barrel, whereas oppositely tuned TC pairs terminate in different parts of the barrel.

\section{Vertical organization of angular preference}

TC neurons that share a particular angular preference converge onto a common region within their corresponding cortical barrel, where multi-unit activities display similar angular tuning. Angular preference is in fact shared by individual neurons within small vertical domains in a barrel. Figure 5 shows peristimulus time histograms and polar plots obtained from two units recorded at depths $100 \mu \mathrm{m}$ apart within a vertical penetration. Although response profiles clearly differed in terms of spontaneous activity and phasic/tonic firing, both cells fired most robustly to caudal $\left(0^{\circ}\right)$ whisker deflections and displayed an overall pref-
A

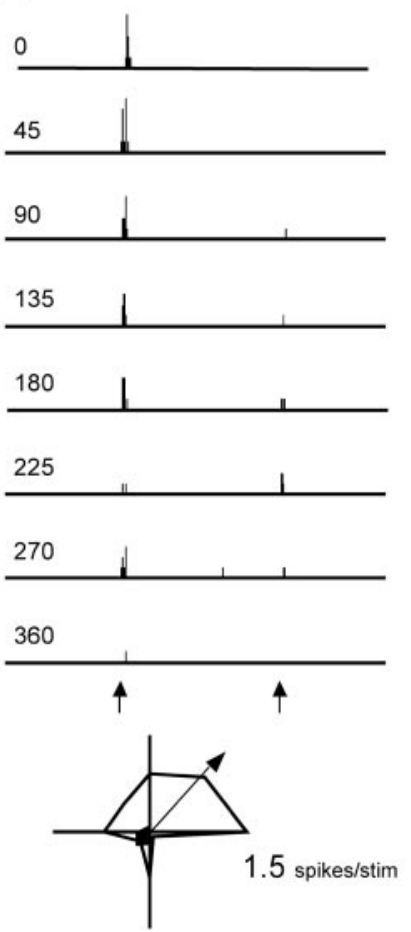

B
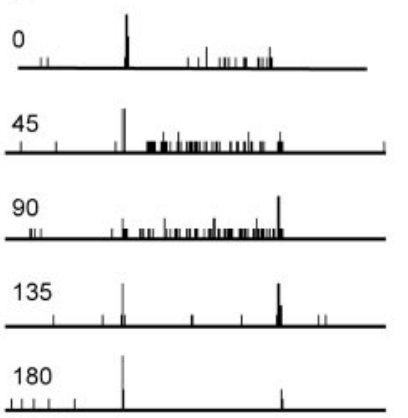

225

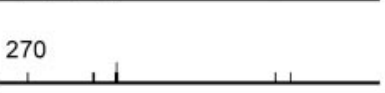

360

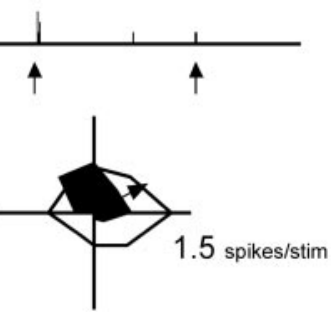

Figure 5. Angular tuning properties of two well-tuned cortical neurons recorded in the same vertical microelectrode penetration within a layer IV barrel. Peristimulus time histograms (PSTHs) in the top panels show accumulated responses of each neuron to 10 whisker deflections at eight different angles (see inset in Fig. 3 for orientation with respect to face). Arrows below the $315^{\circ}$ PSTH denote deflection onset and offset; duration of PSTH $=500 \mathrm{msec}$, and individual ticks represent 1 spike/1 msec bin. Open polar plots at bottom show responses to stimulus onsets plotted in polar coordinates; filled polar plots show responses to stimulus offsets. Both cells fired maximally (1.5 spikes per stimulus) to stimulus onsets in the caudal $\left(0^{\circ}\right)$ direction. Arrows in polar plots indicate average vector angle and strength computed over eight $\mathrm{ON}$ response magnitudes. The unit in $A$ was recorded at a microdrive depth reading of $728 \mu \mathrm{m}$; that in $B$ was at $828 \mu \mathrm{m}$.

erence for the onset of whisker movements (ON responses) in a caudal and dorsal direction (Fig. 5, bottom, unfilled polar plots). The polar plots were similarly shaped, as indicated by the high SI value (0.78), computed as a correlation coefficient across the eight deflection angles. The two units differed with respect to the magnitude and angular tuning of their responses to stimulus offsets (OFF), shown in the filled polar plots.

Polar plot similarity was quantified by computing SIs for the $\mathrm{ON}$ responses of all possible pairs of neurons recorded within the same electrode penetration (Fig. 6C). The average difference in depth was $121 \mu \mathrm{m}$, and the mean SI was $0.21 \pm 0.16(n=330)$. The distribution of values was compared with SIs computed by shuffling the data so that comparisons were made between units recorded in different animals (and thus different penetrations); appropriately, the mean value was close to $0(0.02 \pm 0.14 ; n=$ 188). Mean SIs of "within" penetration and "shuffled" samples differed ( $t$ test; $p<0.0001)$. Similar results were obtained when the analysis was limited to just successively encountered withinpenetration pairs. There was no relationship between polar plot similarity and vertical distance between the location of the recorded units $\left(r^{2}=0.0 ; p=0.95\right)$. Moreover, the SIs of sequentially recorded units separated by $\geq 100 \mu \mathrm{m}(0.23 \pm 0.13)$ were similar to those separated by $<100 \mu \mathrm{m}(0.22 \pm 0.17)$ and larger than those of the shuffled sample. A $100 \mu \mathrm{m}$ separation corresponds to approximately one-half the depth of layer IV. We also 

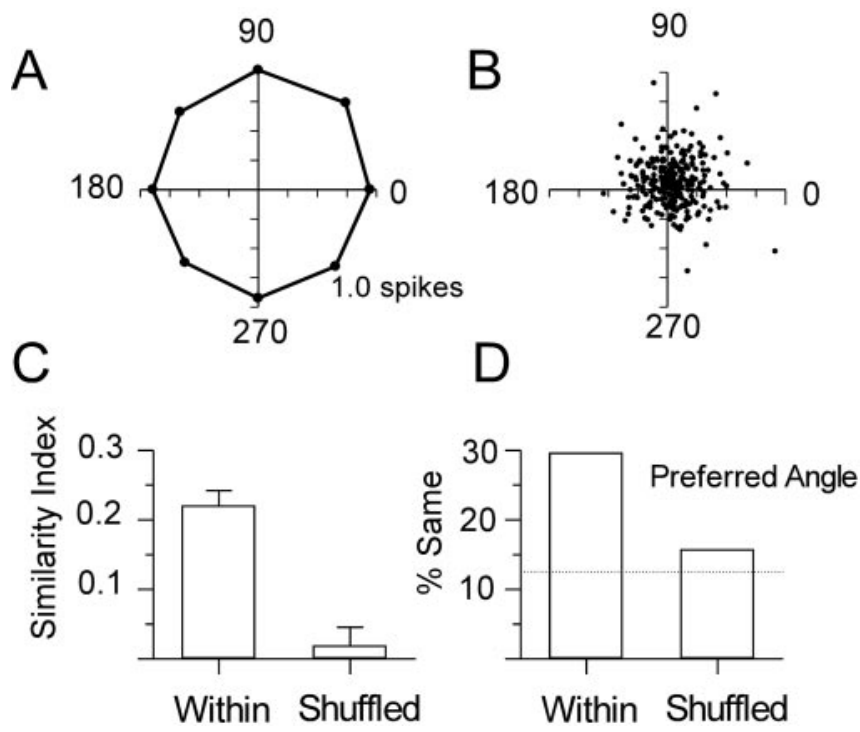

Figure 6. Barrel neurons encountered in the same vertical penetration have a common directional preference. $A$, Average responses of all 250 units at each direction; 1.0 denotes spikes per stimulus onset (see inset in Fig. 3 for orientation). $B$, Angular tuning vectors computed from individual polar plots; the greater the distance from the origin, the more selective the response of the neuron. C, Means \pm SEM. SIs computed for all pairwise comparisons of units recorded within the same microlectrode penetration (Within) and for pairs of neurons recorded in different animals (Shuffled) are shown. An index of 1.0 represents identically shaped polar plots; a value of -1.0 denotes completely dissimilar shapes. $D$, Percentages of within-penetration and shuffled pairs of neurons with exactly the same preferred deflection angle, defined as the angle (of eight) evoking the largest number of spikes per stimulus onset. By chance this would occur $12.5 \%$ of the time (dashed line).

compared polar plots by finding the difference of their mean vectors. Within-penetration polar plots had significantly smaller differences than shuffled comparisons $(63 \pm 49$ SD vs $88 \pm 50$ degree differences; $t$ test; $p<0.001$ ).

In addition to comparing polar plots from different penetrations (i.e., shuffled data), we explicitly examined whether the similarity of polar plots within vertical penetrations was caused by a systematic bias in the preferred angles of the sampled units. All deflection angles were represented in the barrel, as demonstrated by the average polar plot for all recorded neurons (Fig. $6 A$ ) and by the distribution of individual vector angles (Fig. 6B). On average, a deflection in any direction evoked $\sim 1.0$ spike per stimulus. In this sample, but not in others from different studies in our laboratory, a slight bias toward the 0,45 , and $90^{\circ}$ deflection angles was noted (ANOVA; $p<0.01$ ). Therefore, we reexamined similarity indices, excluding any polar plot pair wherein one of the units had an angular preference from 0 to $90^{\circ}$, measured as the vector angle of its polar plot; mean similarity indices still differed significantly from the shuffled comparison group $(p<$ $0.001)$. Differences in mean vectors between within-penetration and shuffled groups also remained robust when similarly examined $(p<0.001)$. Finally, possible within-animal bias was examined by randomly selecting a cell from each penetration and comparing its polar plot with that of a randomly selected cell from a different penetration but the same experiment. This procedure yielded a mean SI of $0.052 \pm 0.38 \mathrm{SD}$ ( $n=374$ comparisons), which is not significantly different from that of our across-experiment shuffled data $(p=0.31)$ but is significantly different from the mean of our within-penetration data $\left(p<10^{-6}\right)$. Thus, our results are unlikely to be caused by any systematic sampling bias.

We also compared polar plots by determining how many unit pairs had identical maximally effective angles, defined as the deflection angle evoking the largest average response to stimulus onset (Fig. $5 A, B, 0^{\circ}$ ). With eight tested angles, this would be expected to occur by chance $12.5 \%$ of the time. Of the 330 withinpenetration pairs, $98(29.6 \%)$ shared exactly the same maximally effective angle (Fig. 6D) $\left(\chi^{2}\right.$ test; $\left.p<0.001\right)$. In some units two or more deflection angles evoked responses that were equivalent and maximal. Because this would bias the probability of finding pairs of polar plots with identical maximal angles, we reanalyzed the within-penetration data, excluding any pair in which one of the polar plots had $>1$ maximal angle. Of the 229 remaining pairs, $54(23.5 \%)$ had exactly the same maximal angle $(p<$ $0.005)$. Finally, $15.7 \%$ of the shuffled pairs had the same maximum angle, a value similar to chance $(p=0.87)$, even when data were not excluded because of the presence of multiple maximal angles.

\section{Multiple angular tuning domains within layer IV barrels}

Neurons within a vertical domain share similar angular preferences. Are such domains represented systematically across the horizontal dimensions of a barrel? To determine this, we first examined data from experiments in which multiple vertical penetrations were made through the same barrel. Once a barrel of interest was identified (see Materials and Methods), small mediolateral or rostrocaudal steps in electrode placement enabled us to make multiple penetrations through the same barrel. Figure 7 shows photomicrographs of a specimen wherein seven vertical electrode penetrations were targeted to barrel C3. Figure $7 \mathrm{~A}$ shows a CO-stained tangential section through cortical layer IV; the box encompasses the region around barrel C3 and is shown at higher magnification in Figure $7 B$. The location of each penetration was verified by reference to surface vasculature drawings, tissue disruption along the track beginning at the pial surface, and the presence of HRP deposits in deeper layers, made in selected penetrations. Six of the seven electrode tracks can be seen in or near barrel C3, two of which were also verified by the presence of small HRP deposits near the layer V/layer VI border (Fig. 7C). In one track (5), subsequently found in the septum between the C3 and D3 barrels and therefore excluded from the analyses, neurons were clearly driven by multiple whiskers (e.g., C3 and D3). Figure 7, $D$ and $E$, shows polar plots obtained from units in two of the vertical penetrations through the barrel center. The two units in penetration 1 both displayed preferences for deflection onsets in the rostroventral direction, as did the units in penetrations 2 and 7 (data not shown). Interposed between penetrations 1 and 7 , units in penetration 3 preferred dorsal deflections. Although data such as these could be interpreted as reflecting, in this case, horizontally interleaved bands of downward versus upward preferring cells, no consistent patterns emerged from the vertical penetrations (see also below). Therefore, we performed two experiments in which we attempted to record across the barrel in the horizontal plane.

Tangential penetrations were used to test for possible systematic representation. Figure 8 shows results from an experiment in which single units were recorded along three tangential penetrations through the B2 barrel. Here, units preferring ventral deflections were located at the posterolateral (units $j$ and $k$ ) and anterolateral corners (units $\mathrm{n}$ and $\mathrm{o}$ ), separated by what appears to be a band of cells with dorsal preferences running through the barrel center (e.g., units c, d, g, l, and m). Similar findings (sequences of common preferred angles that changed abruptly on additional microelectode advancement) were observed in the other experiment using tangential penetrations. 

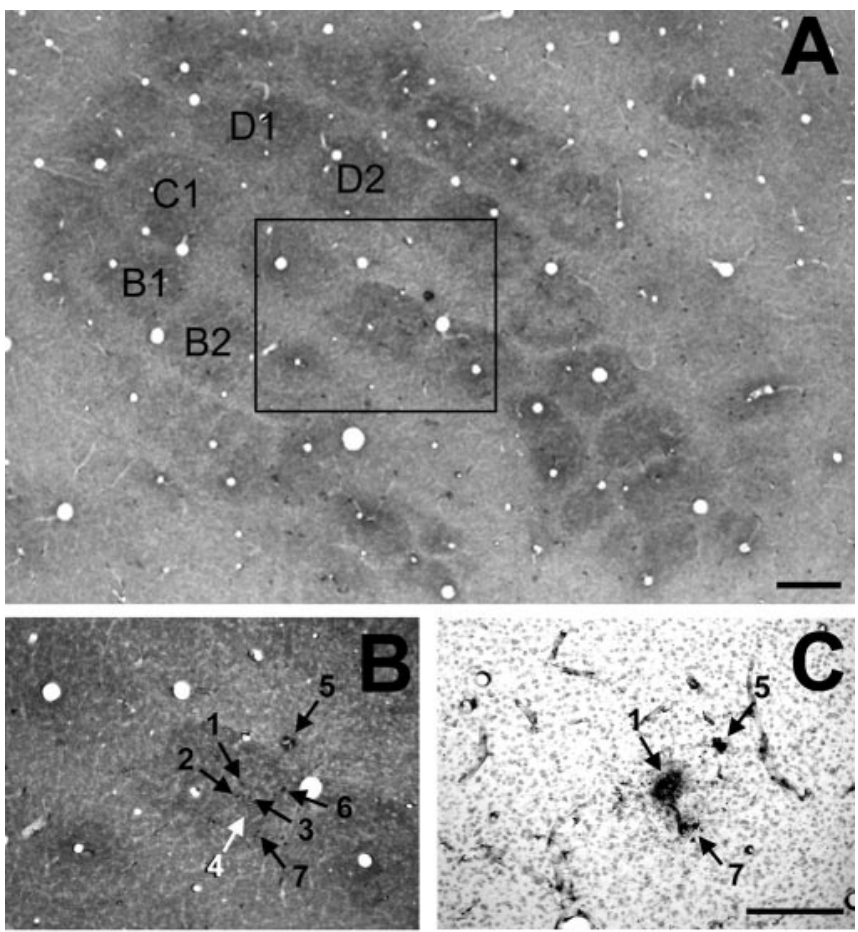

\#1
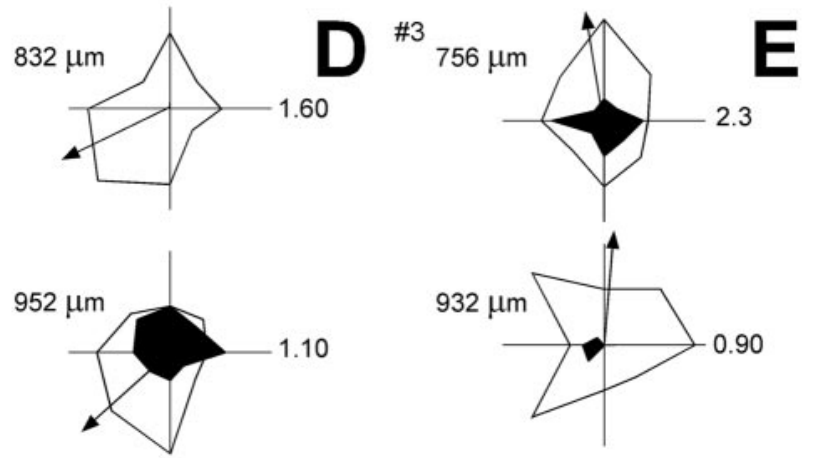

Figure 7. Multiple electrode penetrations in a barrel encounter vertical domains in which successively recorded neurons have similar angular preference but different $0 \mathrm{~N} / 0 \mathrm{FF}$ ratios. $A$, $\mathrm{CO}$-stained tangential section through layer IV in which seven vertical electrode penetrations were targeted toward barrel C3. For orientation, several barrels are indicated by their row and $\operatorname{arc}($ e.g., $\mathrm{B} 1, \mathrm{C}$, etc). The box encompasses barrel $\mathrm{C}$, which is shown at higher magnification in $B$ ). $B$, Higher magnification of boxed area in $A$, showing the location of seven electrode tracks (numbered arrows) targeted to the $(3$ barrel. The entry point of each penetration was recorded on a scaled surface map of the cerebral vasculature. In tangential sections, individual electrode tracks could be followed from their entry point through the superficial layers and into the barrel itself (black arrows). Some tracks were also verified by the location of HRP deposits placed into deeper layers (e.g., $C$ ) or deduced by virtue of their known relationship to identified tracks (white arrow). C, HRP-stained section through layer VI beneath barrel C3; section is radially aligned with that shown in $B$. Numbered arrows indicate HRP deposits made at the termination of penetrations 1 and 7 through barrel $C 3$ are shown. Arrow 5 indicates an electrode track that is also visible in the interbarrel septum in layer IV. Polar plots of the ON (open) and OFF (filled) responses of units recorded in penetrations 1 and 3 are shown. Arrows, drawn at equivalent lengths, indicate mean vectors but not vector magnitude. Numbers to the right of each polar plot indicate scale in spikes per stimulus; the depth in microdrive readings is indicated to the left. See inset in Figure 3 for orientation of polar plots.

We examined whether different preferred angles are represented at fixed locations within barrels, e.g., ventrally tuned cells clustering anterolaterally as in Figure 8, units $j$ and $k$. Each of the 250 units recorded in vertical microelectrode penetrations were assigned to one of nine sectors that subdivided each barrel into $\sim 150-\mu \mathrm{m}$-sided squares (Fig. $8 A$ ). As shown in Figure $9 A$ polar plot vector angles were distributed equivalently across sectors.
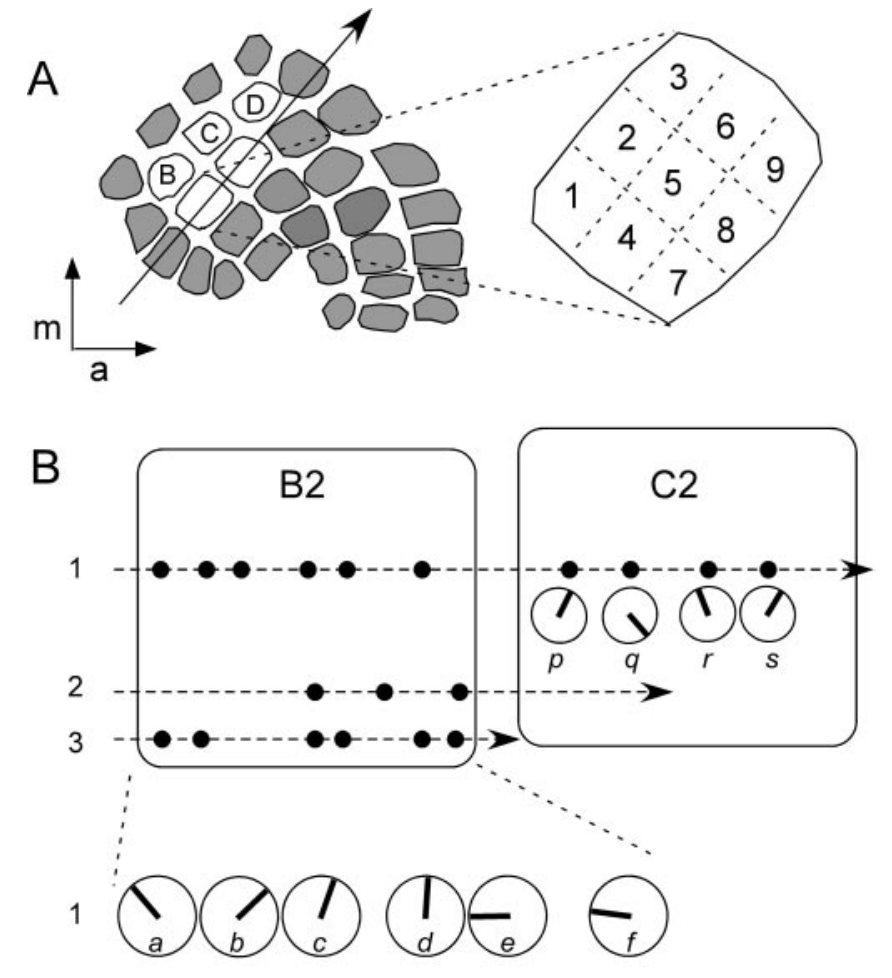

2

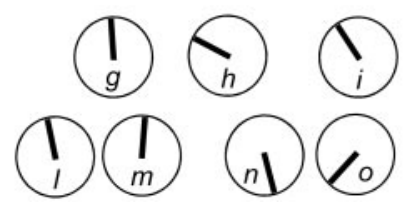

Figure 8. Angular preferences of neurons recorded along tangential penetrations through a barrel change abruptly. $A$, Schematic drawing of a barrel field showing posterolateral to anteromedial trajectory of microelectrode tracks, all of which passed through the $B 2$ barrel. $B-D$ indicate barrels corresponding to the $B 1, C 1$, and $D 1$ whiskers. Each of these barrels is $\sim 400$ $\mu \mathrm{m}$ wide along the axis of the microelectrode track. The expanded barrel to the right illustrates the nine sectors to which individual units were assigned for the analyses in Figure 9; the width of each sector is $\sim 150 \mu \mathrm{m}$. m. Medial; a, anterior. B, Location and angular preferences of all units recorded in three parallel penetrations through $B 2$; penetration 1 also passed through the C 2 barrel. Each solid dot represents a single unit $(\mathrm{a}-\mathrm{s})$, and larger open circles with solid vector lines emanating from their centers indicate angular preferences of the corresponding individual cells; solid lines denote angular vectors, not magnitudes, with $0^{\circ}$ (caudal) to the right and $90^{\circ}$ (dorsal) up. The three lines of circles at the bottom represent units in the three penetrations through B2; the circles in 2 represent the four units recorded in a continuation of track 1.

Mean vector angles varied from 342 to $83^{\circ}$, reflecting the overall caudal/dorsal bias of the sample (see above); virtually identical results were obtained when vector angles were weighted by their magnitudes to increase the contribution of well-tuned cells (data not shown). We interpret these analyses to mean that angular tuning is not represented within a fixed anteroposterior/mediolateral coordinate space that is common to all barrels.

Systematic representations of angular preference, if they exist, could differ from one barrel to the next; therefore, they might be obscured by the pooling of data from different barrels, as in the sector analysis. For example, in the $\mathrm{C} 2$ barrel shown in Figure $8 B$, units with a dorsal preference $(\mathrm{p}, \mathrm{r}, \mathrm{s})$ were separated near the center of the barrel by a ventrally preferring unit (q), a different sequence from that observed in an approximately homologous track in B2 (a-f). Therefore, we examined whether nearby units within the same barrel had similarly shaped polar plots, consistent with the existence of a map of angular tuning within that 

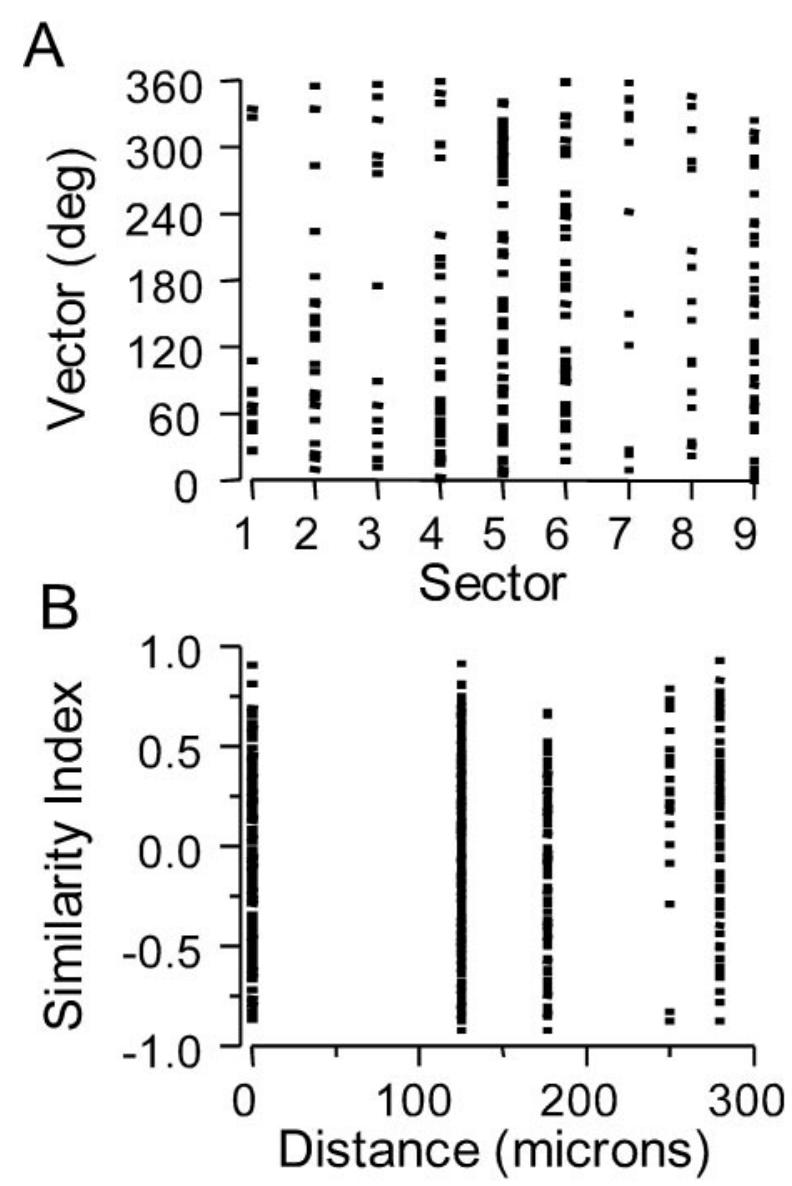

Figure 9. Tangentially separated pairs of neurons are not necessarily likely to be similar or dissimilar. $A$, Polar plot vectors of all units recorded in vertical microelectrode penetrations plotted according to the sector (see Fig. $8 \mathrm{~A}$ ) in which the unit was located. Note that $359^{\circ}$ and $0^{\circ}$ are contiguous. $B$, Sls among pairs of units recorded in different microelectrode penetrations in the same rat. The tangential distance between each pair was taken as the direct distance between the centers of the respective sectors of the unit. 0 indicates that the units were recorded in the same sector in the same rat but in different penetrations.

individual barrel. SIs were calculated for all pairs of units recorded in the same barrel in the same animal (Fig. 9B), but not in the same vertical penetration. The horizontal separation of the units was taken as the distance between the centers of their respective sectors; e.g., 2 units in sector 1 have a spacing of $0 \mu \mathrm{m}$, units in sector 1 and 9 are separated by $280 \mu \mathrm{m}$. Regression analysis revealed no significant relationship between angular preference and unit proximity in the horizontal plane.

Thus, as in the case of the pooled data, angular tuning does not appear to be represented in a rectilinear coordinate system that is unique to each individual barrel.

\section{Vertical organization of $\mathrm{ON}$ and $\mathrm{OFF}$ responses}

We used glass micropipettes for single-unit recording because of their high spatial resolution and their ability to pass through the cortical tissue with minimal disruption and dimpling. During the advancement of the microelectrode, the spike waveform and response characteristics (e.g., magnitude, spontaneous activity, phasic/tonic firing, and ON/OFF characteristics) of each unit were carefully monitored to avoid inadvertently collecting data from the same unit twice. Also, we noted the microdrive depth reading at which an already characterized unit disappeared or fired injury discharges. As a further post hoc control for electrode advancement changing responsiveness of a cell and subsequent classification of the different activity as a new, second cell, we examined the relative sizes of responses to stimulus onset (ON) and offset (OFF) of successively recorded units. OFF responses are disproportionately sensitive to overall cell excitability, as evidenced by the effects of exogenously applied GABA or GABA antagonists (Kyriazi et al., 1996). If, for example, a cell becomes more excitable, then its OFF response becomes disproportionately larger than does its $\mathrm{ON}$ response; the increase in the relative size of the OFF response (OFF/ON ratio) is greater than the relative increase in $\mathrm{ON}$ response magnitude.

We regressed the proportional difference in the size of the $\mathrm{ON}$ responses of successively recorded units with the proportional difference in their OFF/ON ratios. For vertical penetrations, the correlation coefficient was negative $(-0.36 ; p<0.001)$. Thus, on average, if the $\mathrm{ON}$ response increased from one unit to the next, the relative size of the OFF response decreased, and vice versa. The pair of units in Figure 5 displays this characteristic, as does the pair in penetration 1 of Figure 7. This finding is opposite to what one would expect if the same unit was recorded twice but at different levels of excitability. Similar analyses performed for the 31 pairs of successively recorded units in tangential penetrations revealed no relationship between changes in $\mathrm{ON}$ responses and changes in OFF/ON ratios $\left(r^{2}=0.00 ; p=0.87\right)$. Together, the vertical and tangential data indicate that similarities among polar plots of units recorded within a vertical penetration are not an artifact of recording from the same cell multiple times. The inverse correlation observed in vertical penetrations may reflect our attempts to isolate units with clearly different response properties. Nevertheless, the findings demonstrate that neurons in the vertical register, which have the same angular preference, do not necessarily have similar ON/OFF properties.

\section{Discussion}

Here, we investigated in detail functional micro-organization within individual layer IV barrels. LFPs evoked by single thalamic neurons vary across the tangential dimension of a barrel, indicating that individual thalamic neurons have a functional impact on some parts of a barrel more than on others. A barrel is composed of multiple regions, each receiving convergent inputs from thalamic neurons whose angular preferences are similar to each other and to the preferred angle of nearby, vertically aligned cortical neurons. These findings suggest the existence, within individual barrels, of collections of functional minicolumns based on angular preference. Angular tuning domains are established by convergent inputs from TC cells with similar angular preferences, and processing within such domains may depend on local connectivity among vertically aligned barrel neurons.

A variety of studies have demonstrated that individual barrels are indeed composed of multiple structural/functional domains. In the barrels of behaving mice, 2-deoxyglucose labeling reveals zones of high and low uptake that extend vertically into supragranular and infragranular layers (McCasland and Woolsey, 1988). GABA A receptor immunoreactivity and regions of histochemically localized synaptic zinc are distributed within several patches in a barrel (Land et al., 1995; Land and Akhtar, 1999). These patches coincide precisely with the mosaic of irregularly shaped "blotches" of heightened metabolic activity revealed by cytochrome oxidase staining (Land and Simons, 1985). The density of TC terminations in barrel centers also is nonuniform (Woolsey et al., 1975; Bernardo and Woolsey, 1987; Chmielowska et al., 1989), and single TC axons, although arborizing throughout the barrel center, appear to branch exten- 
sively within restricted zones smaller than the barrel (Jensen and Killackey, 1987; Arnold et al., 2001). Although the precise correspondence, if any, between these heterogeneities and angular tuning domains remains unclear, existing evidence supports the notion of multiple functional circuits, or subnetworks, within individual barrels.

\section{Angular tuning domains and TC microcircuitry}

A striking observation is that the angular preference of postsynaptic cortical units is predicted by the preference of a single innervating thalamic neuron and the size of the terminal potential it evokes at that barrel site. Moreover, two thalamic neurons are most likely to produce similarly sized terminal potentials at a given cortical location when they have similar angular tuning. These findings suggest that similarly tuned TC neurons have overlapping domains of functional synapses, whereas oppositely tuned ones tend to innervate different barrel regions. Crosscorrelation analysis of paired single units likewise have suggested that thalamic neurons are more likely to have functional connections onto regular-spike cortical neurons if they have a similar angular preference (Bruno and Simons, 2002). Individual TC spikes typically evoke only subthreshold responses in regularspike barrel neurons (Bruno and Simons, 2002; Porter et al., 2001), necessitating substantial convergence of multiple, similarly tuned thalamic neurons. That multiple TC axons converge onto a common location within a barrel is consistent with anatomical studies showing a fine-grained topography between individual thalamic barreloids and the corresponding cortical barrel (Land et al., 1995).

The axons of individual TC neurons as well as those of their spiny stellate cell targets (Harris and Woolsey, 1983; Petersen and Sakmann, 2000) arborize densely throughout the depth of layer IV. Barrel neurons that lie in the vertical register have similar angular tuning preferences, a finding consistent with the known radial organization of cortical microcircuits in general and of barrel circuits specifically. Our analysis of angular tuning by barrel sector (Fig. 9) suggests an upper limit of 75-100 $\mu \mathrm{m}$ for the narrowest horizontal dimension of an angular tuning domain. Thus, such domains may correspond to vertically organized minicolumns. In a recent review, Mountcastle (2003) proposes that minicolumns form elemental building blocks of larger functional cortical columns, which in whisker/barrel cortex are represented in layer IV by the barrels themselves. Functional domains of minicolumn scale have been described previously in the somatosensory cortex (Favorov and Diamond, 1990), and orientation preferences in cat area 17 change every $50 \mu \mathrm{m}$ in trajectories parallel to the pial surface (Albus, 1975). In barrel cortex, projections between layer IV and II/III are strongest between cells in direct vertical register (Lubke et al., 2000; Feldmeyer et al., 2002). It remains to be determined whether angular tuning domains within layer IV barrels extend into other layers of the cortex.

In the visual cortex, functional domains are organized spatially across the cortical surface (Blasdel and Salama, 1986; Bonhoeffer, 1991). The form and arrangement of these modules can be complex (e.g., orientation pinwheels), reflecting an organization that optimizes the representation of multiple stimulus features within the visuotopic map (Swindale et al., 2000). Similarly, our data suggest that if a map of angular tuning exists in the barrel, it is not a geometrically simple one. Specifically, the representation of angular tuning does not appear to exist within a fixed anteroposterior/mediolateral coordinate space that is constant for all barrels. Moreover, analysis of individual barrels re- vealed no apparent relationship between angular tuning and horizontal distance across the barrel center. Consistent with these findings from vertical penetrations, the angular preferences of neurons recorded successively in tangential microelectrode tracks can change abruptly and unpredictably. Penetrations in either trajectory reveal that, within a barrel, domains tuned to the same angle can be observed hundreds of micrometers apart in the horizontal plane. One possibility is that angular tuning domains randomly cluster within a barrel. Alternatively, each barrel may contain repeated, organized representations whose individual elements are small and complexly interdigitated. Examples include multiple pinwheels or narrow, sinuous parallel sheets. Their exact shapes and arrangement may be constrained by the geometry of the barrel itself (Senft and Woolsey, 1991). Such structures are difficult to discern using microelectrode recordings.

We estimate that an angular tuning domain is no wider than $100 \mu \mathrm{m}$ (Bruno and Simons, 2002; Keller and Carlson, 1999). Thus, the width of these domains is substantially less than the diameter of a barrel and the horizontal extent of its excitatory cellular components. For example, the dendritic trees of spiny stellate neurons measure $\sim 200 \mu \mathrm{m}$ in horizontal span (Lübke et al., 2000; Simons and Woolsey, 1984). TC axons have maximal horizontal spans of approximately the diameter of a barrel (Jensen and Killackey, 1987), although individual arbors may terminate most densely within a restricted zone of $\sim 200 \mu \mathrm{m}$ (Jensen and Killackey, 1987; Arnold, 2001). The substantial overlap of even restricted tufts of individual TC arbors with each other and with multiple spiny stellate cell dendritic and axonal processes indicates that neighboring neurons within the same minicolumn potentially receive a multitude and diversity of excitatory inputs. Nevertheless, the existence within individual barrels of subdivisions revealed by histochemical markers and of angular tuning minicolumns suggests that a barrel center is parceled into functional domains smaller than the connectivity matrix within it.

How is it that angular tuning domains exist at a scale smaller than the cellular anatomy? Several mechanisms could extract angular selectivity from the interaction of comparatively large TC axonal arbors and cortical dendritic trees. Limited convergence of multiple afferent arbors may provide a means for strong spatial and temporal summation of common inputs by regular-spike barrel neurons (Simons and Carvell, 1989). For example, if one thalamic neuron that prefers $0^{\circ}$ deflections and another that prefers $90^{\circ}$ deflections have axonal territories that overlap each other by $50 \%$, their intersection would be spatially smaller than the individual arbors themselves and functionally biased to $45^{\circ}$, assuming linearity of impact (Jagadeesh et al., 1993). The partial overlap of spiny barrel neuron dendrites within the territory of convergent TC inputs could also constrain the size of angular tuning domains. Finally, connections among nearby cortical neurons, both excitatory and inhibitory, could enhance the nonlinearities of regular-spike barrel neurons (Kyriazi and Simons, 1993; Pinto et al., 1996) and hence their selectivity for whisker deflections in different directions.

\section{References}

Adams JC (1981) Heavy metal intensification of DAB-based HRP reaction product. J Histochem Cytochem 29:775.

Albus K (1975) A quantitative study of the projection area of the central and the paracentral visual field in area 17 of the cat. II. The spatial organization of the orientation domain. Exp Brain Res 24:181-202.

Armstrong-James M (1975) The functional status and columnar organization of single cells responding to cutaneous stimulation in neonatal rat somatosensory cortex SI. J Physiol (Lond) 246:501-538.

Arnold PB, Li CX, Waters RS (2001) Thalamocortical arbors extend beyond 
single cortical barrels: an in vivo intracellular tracing study in rat. Exp Brain Res 136:152-168.

Bernardo KL, Woolsey TA (1987) Axonal trajectories between mouse somatosensory thalamus and cortex. J Comp Neurol 258:542-564.

Blasdel GG, Salama G (1986) Voltage sensitive dyes reveal a modular organization in monkey striate cortex. Nature 321:579-585.

Bonhoeffer TGA (1991) Iso-orientation domains in cat visual cortex are arranged in pinwheel-like patterns. Nature 353:429-431.

Bruno RM, Simons DJ (2002) Feedforward mechanisms of excitatory and inhibitory cortical receptive fields. J Neurosci 22:10966-10975.

Chmielowska J, Carvell GE, Simons DJ (1989) Spatial organization of thalamocortical and corticothalamic projection systems in the rat SmI barrel cortex. J Comp Neurol 285:325-338.

Durham D, Woolsey TA (1977) Barrels and columnar cortical organization: evidence from 2-deoxyglucose (2-DG) experiments. Brain Res 137:169-174.

Favorov OV, Diamond ME (1990) Demonstration of discrete place-defined columns-segregates-in the cat SI. J Comp Neurol 298:97-112.

Feldmeyer D, Lubke J, Silver RA, Sakmann B (2002) Synaptic connections between layer 4 spiny neuron layer $2 / 3$ pyramidal cell pairs in juvenile rat barrel cortex: physiology and anatomy of interlaminar signalling within a cortical column. J Physiol (Lond) 538: 803-822.

Harris RM, Woolsey TA (1983) Computer-assisted analyses of barrel neuron axons and their putative synaptic contacts. J Comp Neurol 220:63-79.

Hubel DH, Wiesel TN (1977) Functional architecture of macaque monkey visual cortex. Proc R Soc Lond B Biol Sci 198:1-59.

Jagadeesh B, Wheat HS, Ferster D (1993) Linearity of summation of synaptic potentials underlying direction selectivity in simple cells of the cat visual cortex. Science 262:1901-1904.

Jensen KF, Killackey HP (1987) Terminal arbors of axons projecting to the somatosensory cortex of the adult rat. I. The normal morphology of specific thalamocortical afferents. J Neurosci 7:3529-3542.

Jones EG, Diamond IT (1995) Cerebral cortex, Vol 11, The barrel cortex of rodents. New York: Plenum.

Keller A, Carlson GC (1999) Neonatal whisker clipping alters intracortical, but not thalamocortical projections, in rat barrel cortex. J Comp Neurol 412:83-94.

Kyriazi HT, Simons DJ (1993) Thalamocortical response transformations in simulated whisker barrels. J Neurosci 13:1601-1615.

Kyriazi HT, Carvell GE, Simons DJ (1994) OFF response transformations in the whisker/barrel system. J Neurophysiol 72:392-401.

Kyriazi HT, Carvell GE, Brumberg JC, Simons DJ (1996) Quantitative effects of GABA and bicuculline methiodide on receptive field properties of neurons in real and simulated whisker barrels. J Neurophysiol 75:547-560.

Land PW, Akhtar ND (1999) Experience-dependent alteration of synaptic zinc in rat somatosensory barrel cortex. Somatosens Mot Res 16:139-150.

Land PW, Simons DJ (1985) Cytochrome oxidase staining in the rat SmI barrel cortex. J Comp Neurol 238:225-235.

Land PW, Buffer Jr SA, Yaskosky JD (1995) Barreloids in adult rat thalamus: three dimensional architecture and relationship to somatosensory cortical barrels. J Comp Neurol 355:573-588.
Lichtenstein SH, Carvell GE, Simons DJ (1990) Responses of rat trigeminal ganglion neurons to movements of vibrissae in different directions. Somatosens Mot Res 7:47-65.

Lübke J, Egger V, Sakmann B, Feldmeyer D (2000) Columnar organization of dendrites and axons of single and synaptically coupled excitatory spiny neurons in layer 4 of the rat barrel cortex. J Neurosci 20:5300-5311.

McCasland JS, Woolsey TA (1988) High-resolution 2-deoxyglucose mapping of functional cortical columns in mouse barrel cortex. J Comp Neurol 278:555-569.

Mountcastle VB (1957) Modality and topographic properties of single neurons of cat's somatic sensory cortex. J Neurophysiol 20:403-434.

Mountcastle VB (2003) Introduction: computation in cortical columns. Cereb Cortex 131:2-4.

Petersen CH, Sakmann B (2000) The excitatory neuronal network of rat layer 4 barrel cortex. J Neurosci 20:7579-7586.

Pinto DJ, Brumberg JC, Simons DJ, Ermentrout GB (1996) A quantitative population model of whisker barrels: re-examining the Wilson-Cowan equations. J Comput Neurosci 3:247-264.

Porter JT, Johnson CK, Agmon A (2001) Diverse types of interneurons generate thalamus-evoked feedforward inhibition in the mouse barrel cortex. J Neurosci 21:2699-2710.

Powell TPS, Mountcastle VB (1959) Some aspects of the functional organization of the cortex of the postcentral gyrus of the monkey: a correlation of findings obtained in a single unit analysis with cytoarchitecture. Johns Hopkins Hosp Bull 105:133-162.

Senft SL, Woolsey TA (1991) Mouse barrel cortex viewed as Dirichlet domains. Cereb Cortex 1:348-363.

Simons DJ (1978) Response properties of vibrissa units in the rat SI somatosensory neocortex. J Neurophysiol 41:798-820.

Simons DJ (1983) Multi-whisker stimulation and its effects on vibrissa units in rat SmI barrel cortex. Brain Res 276:178-182.

Simons DJ, Carvell GE (1989) Thalamocortical response transformation in the rat vibrissa/barrel system. J Neurophysiol 61:311-330.

Simons DJ, Land PW (1987) A reliable technique for marking the location of extracellular recording sites using glass micropipettes. Neurosci Lett 81:100-104.

Simons DJ, Woolsey TA (1979) Functional organization in mouse barrel cortex. Brain Res 165:327-332.

Simons DJ, Woolsey TA (1984) Morphology of Golgi-Cox-impregnated barrel neurons in rat SmI cortex. J Comp Neurol 230:119-132.

Swadlow HA, Gusev AG (2000) The influence of single VB thalamocortical impulses on barrel columns of rabbit somatosensory cortex. J Neurophysiol 83:2802-2813.

Swadlow HA, Gusev AG, Bezdudnaya T (2002) Activation of a cortical column by a thalamocortical impulse. J Neurosci 22:7766-7773.

Swindale NV, Shoham D, Grinvald A, Bonhoeffer T, Hubener M (2000) Visual cortex maps are optimized for uniform coverage. Nat Neurosci 3:822-826.

Woolsey TA, Dierker ML, Wann DF (1975) Mouse SmI cortex: Qualitative and quantitative classification of Golgi-impregnated barrel neurons. Proc Natl Acad Sci USA 72:2165-2169. 